\title{
Femmes, genre, révolution
}

Dominique Godineau, Lynn Hunt, Jean-Clément Martin, Anne Verjus et Martine Lapied

\section{(2) OpenEdition}

\section{Journals}

Édition électronique

URL : https://journals.openedition.org/ahrf/11539

DOI : 10.4000/ahrf.11539

ISSN : $1952-403 X$

Éditeur :

Armand Colin, Société des études robespierristes

Édition imprimée

Date de publication : 1 octobre 2009

Pagination : 143-166

ISBN : 978-2-200-92560-4

ISSN : 0003-4436

Référence électronique

Dominique Godineau, Lynn Hunt, Jean-Clément Martin, Anne Verjus et Martine Lapied, « Femmes, genre, révolution », Annales historiques de la Révolution française [En ligne], 358 | octobre-décembre 2009, mis en ligne le 01 octobre 2012, consulté le 22 avril 2022. URL : http://journals.openedition.org/ ahrf/11539; DOl : https://doi.org/10.4000/ahrf.11539 


\title{
FEMMES, GENRE, RÉVOLUTION
}

\author{
Dominique GODINEAU \\ Lynn HUNT \\ Jean-Clément MARTIN \\ Anne VERJUS \\ Martine LAPIED
}

Pour une histoire qui travaille à sortir les femmes de la relative invisibilité dans laquelle elles ont longtemps été reléguées, la Révolution offre un moment d'observation privilégié. Dans les études actuelles sur la Révolution, il ne semble plus possible de faire l'impasse sur la présence des femmes. Mais, l'histoire du genre pousse à dépasser cette visibilité additive qui ne consiste qu'à redonner aux femmes dans le tableau la place quelles avaient occupée mais qui avait été plus ou moins occultée par la mémoire et l'histoire pendant certaines périodes ${ }^{1}$.

Dans un premier temps, la question posée, essentiellement par les historiennes anglo-saxonnes de la gender history, a été celle du refus des droits qui vont de la privation du droit de vote à l'interdiction de défendre la Nation dans l'armée, le 30 avril 1793, et à la fermeture des clubs féminins, le 30 octobre 1793. La Révolution est alors considérée comme la défaite historique des femmes. Ces thèses ont été discutées. Le refus du droit de cité a engendré de multiples travaux qui ont analysé non seulement les raisons de l'exclusion et ses contestations, mais aussi la façon dont elle fonctionne et les réponses que des femmes, affirmant qu'elles appartiennent au Souverain, tentent d'y apporter. Parallèlement, les inter-

(1) Voir Femmes entre ombre et lumière. Recherches sur la visibilité sociale (XVI $-X X^{e}$ siècles), sous la direction de Geneviève Dermenjian, Jacques Guilhaumou, Martine Lapied, Publisud, 2000. 
rogations sur la question des femmes se sont étendues aux différents champs de la Révolution.

Les recherches actuelles voient se côtoyer, avec profit, démarches théoriques et empiriques. L'approche comparative hommes/femmes apparaît essentielle pour les interprétations. Elle permet de comprendre l'action féminine intégrée dans le mouvement général et de s'interroger sur d'éventuelles voies féminines de pratiques politiques.

Le questionnement des archives met en visibilité des modes d'action politique des femmes, montre le sens de leurs engagements. Ces actions peuvent être analysées de façon processuelle, dans leur déroulement, leur temporalité propre et leurs interactions. On saisit mieux pourquoi et comment se font les mutations qui dérèglent l'ordre établi à un moment donné ${ }^{2}$.

Une réflexion problématisée en fonction de la question du genre permet de confronter fructueusement les éléments issus des enquêtes de terrain aux représentations telles qu'elles se manifestent dans les témoignages des contemporains, l'historiographie, la littérature, l'art.

La rencontre entre histoire du genre et histoire de la Révolution s'insère dans le mouvement d'élargissement des champs de réflexion sur la Révolution, élargissement nécessaire si son histoire veut rester au carrefour des recherches ${ }^{3}$. Dans une mise au point sur les récents travaux sur la Révolution française, Suzanne Desan, qui y discerne un abandon des schémas d'analyse de classes, hérités du structuralisme, montre combien, aux États Unis, la réflexion sur le social est stimulée par le féminisme, le multiculturalisme et le débat sur la construction culturelle des identités sociales dans une perspective d'historicisation intégrale, donc prenant en compte le statut de la féminité, de la domination masculine et de la différence de sexes ${ }^{4}$.

(2) Ainsi, le colloque de Rennes sur Le genre face aux mutations consacrait-il une partie à «Pouvoirs et Révolution» où cinq communications portaient sur la Révolution française (Le genre face aux mutations. Masculin et féminin du Moyen-Âge à nos jours, sous la direction de Luc CAPDEvila, Sophie Cassagnes, Martine Cocaud, Dominique Godineau, François Rouquet, Jacqueline SAINCLIVIER, Rennes, Presses Universitaires de Rennes, Collection Histoire, 2003).

(3) Voir Martine Lapied et Christine PEYRARd (dir), La Révolution française au carrefour des recherches, Publications de l'Université de Provence, Collection Le temps de l'histoire, 2003 et Jean-Clément Martin (dir), La Révolution à l'œuvre. Perspectives actuelles dans l'histoire de la Révolution française, Rennes, Presses Universitaires de Rennes, Collection « Histoire », 2005.

(4) Voir Judith Butler, Trouble dans le genre. Pour un féminisme de la subversion, Paris, La Découverte, 2005. 
Dans l'historiographie révolutionnaire, le champ de l'histoire du gender est devenu un champ crucial du questionnement méthodologique sur le nœud politique, société, culture 5 .

Les regards croisés que nous proposons ici nous permettent de bénéficier de différentes approches dans un espace d'expérimentation ouvert. Nous avons interrogé ${ }^{\text {: }}$

Dominique Godineau dont les recherches ont montré la richesse des actions concrètes et spécifiques menées par des femmes et ont mis en valeur l'engagement des Parisiennes qui les place au cœur de certains des événements fondamentaux de la Révolution?

Lynn Hunt dont le roman familial de la Révolution française, placé sous le signe de la psychanalyse, a fait date ${ }^{8}$.

Anne Verjus qui a avancé, dans ses travaux sur la sphère domestique, l'hypothèse selon laquelle les femmes sont mises à l'écart de la participation politique sans, à proprement parler, être exclues de la représentation politique?

Jean-Clément Martin, auteur d'une toute nouvelle synthèse sur « la révolte brisée » où il a souhaité interroger «toutes les dimensions de la vie dans lesquelles les questions sexuées jouent $»^{10}$.

Essayer de comprendre ce qui s'est débattu autour des « citoyennes » remet en cause bien des certitudes, conduit à de nouvelles interrogations qui peuvent aboutir à remanier la vision générale de l'édifice social. C'est toute la richesse de cette voie de permettre plusieurs types d'approches dans la compréhension de la Révolution. Dans ce domaine, encore largement en friche, le chemin est ouvert pour d'autres réflexions et recherches que nous espérons stimuler par ces échanges.

(5) Suzanne Desan « What's after Political Culture ? Recent French Revolutioary historiography », dans French Historical Studies, vol. 23, n 1, 2000.

(6) Je remercie Jacques Guilhaumou pour ses suggestions dans l'élaboration des questions.

(7) Voir Dominique Godineau, Citoyennes tricoteuses. Les femmes du peuple à Paris pendant la Révolution française, Alinéa, 1988, réed. Perrin, 2004 ; ainsi que les études nombreuses, et toujours éclairantes, publiées depuis cet ouvrage fondamental.

(8) Lynn Hunt, The Family Romance of French Revolution, Berkeley, University of California Press, 1992 ; traduction française : Le roman familial de la Révolution française, Paris, Albin Michel, 1995.

(9) Anne Veruus, Le cens de la famille. Les femmes et le vote, 1789-1848, Paris, Belin, 2002.

(10) Jean-Clément Martin, La révolte brisée. Femmes dans la Révolution française et l'Empire, Armand Colin, Paris, 2008. 


\section{Martine LAPIED}

Avec les progrès, indéniables, de la visibilité des femmes dans la Révolution française, peut-on dire que "le combat historiographique " est gagné ou en cours? L'histoire du genre est-elle vraiment reconnue comme une grille de lecture de la Révolution française ou s'agit-il seulement d'une visibilité additive et non du lieu de structuration d'un champ problématique?

\section{Dominique Godineau}

Si combat historiographique il y a, il a en partie été gagné et s'est en partie déplacé. Ce champ de la recherche a ainsi acquis une (relative) reconnaissance scientifique, ce qui était loin d'être évident il y a $30 \mathrm{ou}$ même 20 ans. Aujourd'hui, travailler sur les femmes et/ou sur le genre pendant la Révolution apparaît comme un «vrai » sujet, pris en compte dans plusieurs synthèses récentes ( $c f$. en particulier celles parues pour le CAPES et l'agrégation en 2004-2006). Et les bilans historiographiques n'ignorent pas ces travaux, comme en témoignent les deux colloques d'Aix-en-Provence (2001) et Paris (2004). Notre savoir s'est enrichi, nos thématiques ont été renouvelées, notamment par la problématique du genre (facteur essentiel de la construction et du fonctionnement d'une société, et de sa réinvention pendant la Révolution), que les jeunes chercheurs manient avec familiarité. Cet optimisme doit cependant être modéré.

Tout d'abord parce que cet axe de la recherche ne figure pas toujours dans les ouvrages généraux sur la Révolution. Et, même en considérant que le «combat» de la visibilité est plus ou moins en passe d'être " gagné », on ne peut en dire autant de ce qui concerne l'aspiration à en faire une des grilles de lecture de l'histoire de la Révolution, et pas un simple additif.

Ce qui pose la question de l'articulation de l'histoire des femmes et du genre avec le récit historique sur la Révolution. En effet, ces recherches sont avant tout une façon d'enrichir l'histoire de la Révolution : prendre en compte sa dimension sexuée (ou genrée) permet non seulement de compléter nos connaissances mais aussi, et surtout, de déplacer le regard, d'éclairer autrement l'épisode révolutionnaire, et de faire ainsi surgir de nouvelles problématiques. Aussi, même si cela est encore nécessaire, il ne suffit pas d'indiquer que « les femmes aussi » ont (ou n'ont pas) participé et contribué à la Révolution, à tel épisode, à tel mouvement (politique, social, culturel...). Il ne suffit pas non plus de mettre en évidence le poids du facteur genre. Il faut aussi questionner l'histoire de la Révolution pour tenter de dégager l'articulation entre le rapport de genre et l'événement 
révolutionnaire. Les militantes parisiennes n'étaient, par exemple, pas « à côté » du mouvement populaire, elles en formaient une des composantes structurelles, mais, n'ayant ni la même place, ni les mêmes droits, ni donc les mêmes possibilités d'intervention que les militants, elles élaboraient avec ceux-ci des relations contrastées, qui ne furent pas sans influence sur la forme et le déroulement de certains épisodes.

\section{Jean-Clément MARTIN}

Appliquée à la Révolution française, l'histoire des femmes et du genre demeure encore d'un emploi limité et ambigu en France. Malgré des travaux connus, il est toujours nécessaire de justifier ce qui apparaît comme un point de vue parmi d'autres, une « dimension » surajoutée. La bibliographie reste limitée à quelques dizaines de titres en français, et l'ouverture aux ouvrages étrangers est restreinte. Cette situation est paradoxale, oubliant à quel point l'historiographie française de la Révolution française du XIX ${ }^{\mathrm{e}}$ siècle a été sensible à la place des femmes. Le XX $\mathrm{XX}^{\mathrm{e}}$ siècle a représenté, jusque dans les années 1980, une régression notable.

\section{Anne VerJus}

Il faut bien dire que, depuis le milieu des années 2000, hormis le travail synthétique de Jean-Clément Martin, on n'a guère publié, en France, sur les femmes ou sur le genre en Révolution. Quelques thèses, cependant, montrent que l'intérêt reste entier chez quelques étudiant.e.s ou jeunes docteur.e.s, quoique probablement peu encouragé.e.s (Karine Lambert sur les conflits conjugaux; Laura Talamante sur les femmes de Marseille ; Yann-Arzel Marc sur la citoyenneté ; Guillaume Mazeau sur Charlotte Corday). C'est vers les États-Unis, où les études « genre » ont permis la mise en place de recherches soutenues sur l'histoire des femmes, qu'il faut se tourner pour se faire une idée des directions que prend désormais la recherche sur cette question. On peut dire que, dans les grandes lignes, on assiste à une remise en question des paradigmes sexualistes qui avaient initié le renouveau de l'histoire des femmes : on étudie Rousseau, par exemple, moins comme le misogyne qui a cherché à enfermer les femmes que comme l'auteur qui, par La Nouvelle Héloïse et la promotion de la conjugalité, a permis l'accès des femmes à l'égalité (thèse de Jennifer J. Popiel); les veuves sont à l'honneur également, dans une approche raisonnablement optimiste des pouvoirs que le droit laissait aux femmes à la mort du chef de famille (Janine Lanza); enfin, c'est une approche compréhensive du regard que Napoléon posait sur les femmes qui a guidé le travail de June K. Burton. 
Une approche cantonnée à la radicalité et aux personnalités dites féministes a été très explorée et touche peut-être, du moins en l'état actuel du matériau connu, à ses limites : la rareté relative de ce matériau laisse penser que les révolutionnaires sont aussi pro-femmes que nous sommes pro-enfants aujourd'hui. La «domination masculine», quoique patente, n'est pas problématisée en tant que telle : mise en acte comme un fait « de nature ", elle est quasiment inquestionnable dans le champ politique. Je pense qu'on n'avancera plus tant qu'on n'aura pas accepté d'appréhender l'histoire des hommes et des femmes selon les catégories à partir desquelles eux-mêmes, dans leur grande majorité, se pensaient - et pensaient le monde. Je pense qu'en France, c'est de ce déficit que nous souffrons ; et qu'il explique en grande partie le peu d'estime dont, hélas, on crédite l'histoire des femmes dans les autres disciplines. Je pense qu'il faut travailler, ensemble, à contextualiser - et donc à distinguer et qualifier différemment selon les époques et les lieux - ce qu'on a eu tendance à poser comme un bloc an-historique, à savoir la domination des hommes sur les femmes.

\section{Lynn HUNT ${ }^{11}$}

Le combat historiographique n'est pas encore définitivement gagné. Les femmes sont plus visibles mais leur importance n'est pas toujours reconnue. Malgré tout, de bien des façons, l'attention portée aux femmes, au genre et à la famille a subtilement transformé le paysage historiographique en l'ouvrant autant que faire se peut, et en sapant toutes ces interprétations qui essayaient de minimiser l'impact de la Révolution. Les études sur les femmes et le genre ont montré que les enjeux révolutionnaires étaient fondamentaux dès le départ, non parce qu'ils concernaient le mode de production selon une stricte interprétation marxiste (capitalisme versus féodalisme), mais parce qu'ils concernaient la nature de l'ordre social, et que la place des femmes et de la famille était centrale pour redéfinir ce dernier. Aux privilèges et à la position sociale, définis par la naissance ou les statuts corporatifs, se substituaient les droits de l'individu, réduits par le pouvoir patriarcal, et les carrières s'ouvraient aux talents. La traditionnelle alliance entre le trône et l'autel était supplantée par la sécularisation de l'état-civil et la participation populaire aux événements politiques et religieux. Loin de venir d'en-haut, ces changements étaient portés par la base, et les femmes jouaient un rôle

(11) La traduction des réponses de Lynn Hunt a été assurée par Philippe Bourdin. 
majeur pour les rendre effectifs (ou leur résister). De plus, l'accent mis sur l'autonomie individuelle (le droit de choisir son conjoint, son métier, et de participer à la vie politique) soulevait inévitablement des questions sur celle des femmes ou sur leur dépendance. La promotion des talents amenait de la même façon à s'interroger sur ceux des femmes, leur éducation, leurs perspectives d'avenir, comme Sieyès fut l'un des premiers à le reconnaître. La famille était confrontée à de nouvelles pressions : l'union maritale n'était plus nécessairement nouée ad vitam aeternam, et ne relevait plus des mêmes types de réseaux communautaires et corporatistes. Aux mères incombaient les nouvelles responsabilités de reproduire l'ordre social et politique (et maintenant biologique) ; les sœurs pouvaient désormais ester en justice pour défendre leurs droits d'héritage, etc... L'univers était sans dessus dessous, non seulement sur le plan politique (une république en lieu et place d'une monarchie) mais aussi sur celui des individus et des familles.

L'essentiel du débat historiographique des années 1960 et 1970 porta sur les acteurs de la Révolution : fut-elle une révolution de la bourgeoisie contre l'aristocratie, une coalition de classe (Lefebvre), ou le fait de notables (Eisenstein, Taylor, Cobban, Furet) ? Parce que les critiques anglo-américains et leurs alliés français (Furet) étaient obnubilés par la question du marxisme, ils avaient tendance à jeter le bébé Révolution avec l'eau du bain de ce dernier. Dans les années 1980, qui voient le succès de la critique furétienne, la Révolution devient le malencontreux « circuit sémiotique » dans lequel parler pour le peuple remplace toute réelle activité politique. Déjà dans cette décennie pourtant, et davantage encore depuis, les historiens des femmes et du genre étaient au premier rang pour démontrer que le discours national ne déterminait pas les jeux de pouvoir. Les femmes marchaient, pétitionnaient, cachaient les prêtres ou les dénonçaient, participaient aux contestations des propriétés, divorçaient: de mille façons et par des millions d'actes individuels elles prouvaient que le pouvoir réel venait d'en bas, c'est-à-dire qu'il était juste une forme d'acte politique parmi d'autres, et que la Révolution était l'apprentissage d'une démocratie large et diffuse et non simplement le prolongement inattendu d'un pouvoir absolu centralisé. L'histoire des femmes et du genre peut en conséquence être appréhendée comme la voie vers une réinterprétation majeure de la Révolution, selon laquelle la mutation de la société ne découlerait pas d'un conflit de classes (qui, au demeurant, n'est pas hors de propos) mais bien plutôt d'une reconfiguration par la base des réseaux sociaux et du pouvoir politique. Les formes traditionnelles d'intervention (émeutes de subsistances, pétitions) pren- 
nent alors une toute autre signification, et surgissent de nouvelles façons d'agir, avec ou sans intercession de dirigeants engagés (manifestations, contrôle laïc des cérémonies catholiques, procès pour divorce et héritage, combat pour un état civil des naissances et des mariages - particulièrement dans les colonies -, participation aux clubs). Il ne s'agit pas, comme chez Foucault, d'un pouvoir diffusé par capillarité partout dans la société avec les nouvelles techniques disciplinaires éprouvées au sein des différentes institutions à caractère carcéral (prisons, mais aussi écoles, entreprises, garnisons) ; il s'agit d'un pouvoir saisi et remodelé par des millions d'individus, parfois par leur action individuelle, le plus souvent par leur action collective.

\section{Martine LAPIED}

Nombre de débats, passés et actuels, partent d'un récit de la «fermeture » qui est remis en cause par certains travaux centrés sur un récit d'"ouverture ». Démocratie exclusive des femmes, construction d'une société machiste sans nul doute. Mais où situez-vous alors la question des droits politiques dans le trajet de l'exclusion du politique au nom de la nature à la valorisation de la place de la mère dans une vue familialiste et unifiante de la politique?

\section{Jean-Clément MARTIN}

Malgré « l'invisibilité » coutumière des femmes dans les études historiques sur la période, on ne peut plus ignorer leurs places et leurs rôles. Leur participation, à un titre ou un autre, dans la vie politique publique, y compris dans les élections de 1789 à 1793, n'est pas contestable. Dans le camp révolutionnaire ou contre-révolutionnaire (celui-ci nettement moins considéré par les analyses), les engagements féminins ont été réguliers, constants, nombreux, et faut-il le souligner déterminants, que ce soit en juin 1792, au printemps 1795 , et dans les résistances à la Révolution de 1791 à 1793. « L'ouverture » de l'espace public aux femmes est une réalité pendant la période, même si la « fermeture » est nette après l'automne 1793. Les conséquences de ce moment seront durables, permettant l'enracinement de pratiques féminines dans la vie politique - au sens large - de la France du XIX ${ }^{\mathrm{e}}$ siècle.

En se cantonnant à la prise en compte des «femmes » comme catégorie, la période révolutionnaire pourrait être comprise ainsi. La structure par «ordres » de la société organiciste est remplacée par une société fondée sur l'utilitarisme et la spécialisation des fonctions et des positions de pouvoir. Dans cette redéfinition des groupes sociaux, les 
femmes nobles voient leur prestige, lié à la naissance, affaibli, voire nié, exception faite dans les rangs de la contre-révolution. Globalement, les « femmes » sont, comme les « esclaves » ou les « pauvres », rangées hors de la « grande politique ». Relevons que, au même moment, les spécialisations professionnelles et sociales deviennent déterminantes, créant des groupes plus ou moins éloignés de la vie collective, mais que les exclus de la politique investissent le champ du religieux et du social (pensons aux révoltes) pour continuer à intervenir autrement. Prendre en compte cette répartition remet en cause les analyses régulièrement faites sur la « politique » réduite aux débats, aux manifestations et aux symboles, et permet de lire autrement les émotions, les « baccanales » et de comprendre qu'au XIX ${ }^{\mathrm{e}}$ siècle, les mouvements socialistes et légitimistes aient compté dans leurs rangs des hommes comme des femmes.

\section{Dominique Godineau}

Les débats sur les droits politiques des femmes, sur leur exclusion ou non de l'espace politique et de la cité, me semblent fondamentaux car non seulement ils interrogent en filigrane la nature de la Révolution française, mais renvoient aussi à des approches diverses de l'histoire des femmes et du genre, et de ses méthodes. Ainsi, si certains travaux dessinent plutôt un récit « d'ouverture », c'est en grande partie parce qu'ils ne se fondent pas uniquement sur l'analyse des discours normatifs (ceux des dirigeants révolutionnaires, qui construisent effectivement le plus souvent un "récit de fermeture ») mais aussi sur les archives, et donc sur les activités des individus. Cette démarche consiste à envisager les femmes comme des sujets historiques, des «actrices» ayant contribué à écrire l'histoire, et pas seulement comme des victimes de la « misogynie masculine ». Que l'on comprenne bien : il ne s'agit pas par là d'opposer discours et pratiques, représentations et « réel», études théoriques et études « de terrain », mais de plaider pour une histoire qui mette en résonance et en tension ces différentes composantes. Une histoire qui conjugue exclusion et participation féminine, qui interroge leurs mécanismes tout en secouant leurs évidences parfois trompeuses. Une histoire qui rappelle que les interventions des individus dans le tissu social et politique peuvent brouiller, déplacer les cadres dans lesquels on souhaiterait les tenir enfermés, tout en n'oubliant pas que ces cadres pèsent sur les possibilités et les modalités de leurs interventions.

La question des droits politiques peut être abordée sous cet angle d'approche. Aux discours justifiant l'exclusion du politique au nom de la nature radicalement différente des deux sexes, ou à ceux présentant une 
vue familialiste de la politique, on peut ajouter celui, pas moins important, de l'égalité des droits. Il part de deux postulats : par-delà des fonctions sociales différentes, hommes et femmes sont de même nature («qu'y a-t-il de commun entre vous et nous ? tout... » écrit Olympe de Gouges), ils appartiennent à « la même espèce »; or, selon les principes du droit naturel déclarés par les révolutionnaires, tous les êtres humains possèdent par naissance les mêmes droits. Il n'est ainsi pas dit pendant la Révolution que les femmes sont exclues des droits politiques du citoyen, mais qu'elles ne peuvent les exercer. La différence n'est pas nulle, et certain(e)s ont su l'utiliser, demandant qu'on « rende » leurs droits aux femmes. Les partisans de l'égalité mettent alors en avant les principes et soulignent que, dans l'espace de réciprocité ouvert par la Révolution, les méconnaître conduit à détruire les fondements de la société en construction $-c f$. Condorcet : «Ou aucun individu de l'espèce humaine n'a de véritables droits, ou tous ont les mêmes...» et Guyomar : ou les droits sont les mêmes « ou bien l'immortelle déclaration contient une mortelle exclusion $»$.

Mais, comme nous l'avons dit, la question des droits politiques n'est pas limitée aux textes théoriques. Dans les pratiques elle rencontre celle de la citoyenneté (qui, il faut le souligner, n'est pas alors une notion fermée et figée, mais qui se construit à travers les événements) et de la souveraineté. Or les femmes sont appelées citoyennes et sont membres du souverain. Ce qui permet un « jeu » entre corps social et corps politique, dont le pivot est le mot citoyenne.

\section{Lynn Hunt}

La question de la conscience politique est cruciale, mais il est important de comprendre comment elle est devenue pertinente. De ce point de vue, mon opinion est la suivante :

1) Il y a une dégradation du pouvoir de l'État central, qui a pour cause l'incapacité de la monarchie à répondre aux défis lancés par une opinion publique de plus en plus bruyante - elle reflète pour une part l'ascension de la bourgeoisie.

2) La dégénérescence du pouvoir central ouvre la porte aux incertitudes politiques, et d'ordinaire dans ces situations le peuple saisit l'opportunité de développer d'abord les formes traditionnelles de son activité politique, avant d'en inventer d'autres. Cette créativité est moins « féminine » que populaire, et ressort essentiellement d'initiatives collectives. L'action naît sans beaucoup de préméditation ou de claires intentions (la marche sur Versailles en étant l'un des premiers exemples). Mais une fois 
apparues ces formes nouvelles d'expression politique, des leaders nationaux, des militants locaux, le petit peuple doivent leur donner sens.

Les élites ne contrôlent pas ce processus d'objectivation. Chacun profite des nouvelles formes de la conscience politique, et chaque nouvelle série d'actions entraîne de nouvelles interprétations. Ainsi, une fois advenue une rupture, particulièrement après le 20 juin et le 14 juillet 1789, les députés adoptent la Déclaration des droits de l'homme (une première étape dans la prise de conscience), qui les incite à un tournant dans leurs réflexions et leurs élaborations politiques (les étapes suivantes). Les femmes, comme les protestants, les juifs, les hommes de couleur, et les hommes dépourvus de biens fonciers acquièrent tous une compréhension neuve de leur propre situation et agissent en conséquence. Les droits politiques naissent de ce processus et ne sont jamais définitivement fixés puisque ledit processus n'est jamais complètement achevé. L'ouverture et la fermeture, les droits individuels et les droits exercés au sein de la famille, sont en même temps à l'ordre du jour, et le demeurent pour au moins les deux siècles qui suivent.

Le « processus » se résout en une série de négociations entre gens ordinaires et autorités, entre militants et leurs partisans, entre responsables nationaux et locaux, etc... Ce qui est révolutionnaire dans ces négociations, c'est leur instabilité et l'extension de leur champ ; rien ne va de soi. La prise de conscience politique tient en la demande d'explications : pourquoi les femmes n'obtiennent pas le droit de vote? La question, en quelque sorte, est plus significative que les réponses. L'exclusion du politique au nom de la nature, ainsi que la valorisation de la place de la mère dans une vue familialiste et unifiante de la politique sont toutes deux les réponses à la même question : pourquoi les femmes n'obtiennent pas le droit de vote ? Cette question ne peut être résolue aussi longtemps que voter n'est pas un droit. Quand élire est un privilège accordé aux membres d'une corporation ou d'un corps, une telle absence ne soulève pas de questions (vous n'en faites simplement pas partie). Quand il s'agit au contraire d'un droit reconnu à tous les citoyens « actifs », alors la définition de cet adjectif devient lourde de conséquences et le contrôle sémantique crucial. Par conséquent, la conscience politique tient à la faculté de comprendre qu'il y a là un problème à soulever et que les solutions ne sont pas pré-établies.

\section{Anne VerJus}

La question par laquelle a débuté le renouveau de l'histoire politique des femmes en France, à la fin des années 1980, a été celle-ci : 
pourquoi les femmes, qui sont des Hommes comme les autres, n'ont-elles pas bénéficié des mêmes droits que les « hommes » ? Pourquoi n'ont-elles jamais obtenu le droit de participer aux élections ?

Les historien.ne.s ont tout d'abord mis en avant la chronologie : en 1789, la Déclaration des droits de l'homme ne fait pas mention de la différence de sexe. L'Homme est donc l'Être humain ; qui plus est, la Constitution de 1791 fait du mariage un contrat unissant deux individus libres et égaux en droits. On peut donc en déduire logiquement que les législateurs considèrent les hommes et les femmes comme des individus égaux. Pourtant, les femmes ne sont pas admises à voter; et dès 1793, les femmes se voient interdire explicitement la réunion en club.

L'explication de cette interdiction résiderait dans la conjoncture de cette année 1793 qui voit les femmes envahir les sections, la rue, l'Assemblée, et déborder les hommes politiques. Ceux-ci auraient alors pris la décision de se protéger, et de ne plus admettre les femmes parmi eux. Les hommes se sont donc, selon cette interprétation, ligués contre les femmes en tant que classe de sexe dominée, ou plutôt, ici, vaincue à partir de 1793. Toute une série de travaux, notamment en histoire du droit, ont convergé vers cette interprétation au moment du bicentenaire : je pense aux articles bien connus d'Elisabeth Guibert-Sledziewski ou de Vida Azimi.

Cette interprétation suppose que les femmes ont été jusque-là une classe en position de négocier et d'obtenir la reconnaissance d'un droit de suffrage pour ses membres, ce qui n'a, jusqu'à maintenant, pas été démontré. Dominique Godineau a, au contraire, montré qu'elles avaient très largement dédaigné cette revendication-là ; quant à déterminer si les citoyennes agissaient en tant que membres d'un groupe se définissant de manière distincte voire antagoniste du groupe des hommes, c'est un travail qui reste à faire, permettant le cas échéant d'identifier les premières traces d'une conscience de classe de sexe chez les uns et les autres (à la manière dont William Sewell avait travaillé sur la construction de la conscience de classe ouvrière dans les années 1830).

Cette interprétation suppose également que les hommes auraient inclus les femmes dans la Déclaration des droits de l'homme pour revenir, une fois la Terreur installée, sur leur position ; or, 1793 ne marque pas de césure dans le droit politique des femmes ; c'est dès 1789 qu'elles sont hors de la participation électorale. Dès lors, on peut difficilement faire des discours de Chaumette et Amar le point de rupture dans une Révolution qui se serait d'abord pensée comme émancipatrice des femmes en tant que telles. Les travaux sur le droit du divorce laissent penser au contraire 
que la libéralisation du mariage est moins portée par un discours « féministe » qu'individualiste - et que c'est en particulier en raison des bénéfices inattendus que les femmes ont retirés du divorce pour incompatibilité d'humeur que cette forme-là a disparu du droit civil après Thermidor.

Une autre interprétation a consisté à poser la contradiction comme interne, fonctionnelle et, finalement, constitutive de la démocratie. On a alors parlé, avec Carole Pateman et Geneviève Fraisse, de démocratie exclusive. Le principe est le suivant : la Déclaration des droits de l'homme a d'emblée été pensée à partir de la catégorie d'un homme de sexe masculin; toute la démocratie repose sur la différence de sexe qui en est le principe constitutif, car pour penser l'égalité là où régnait la diversité (notamment celle des trois ordres), rien n'était plus efficace que l'établissement de frontières; l'altérité, incarnée par le sexe faible, a servi cette cause. La Déclaration des droits de l'homme a été faite par les hommes, pour les hommes. Ce type de recherche, aussi novateur que fructueux, recélait plusieurs problèmes. Le principal de ces problèmes était de partir d'une proposition contradictoire, à savoir : que l'universalisme est philosophiquement sexiste. Or, ou bien c'est l'un, ou bien c'est l'autre : soit les révolutionnaires sont sexistes et dans ce cas l'universalisme n'est qu'une gangue vide, il n'est pas universel ; soit ils sont universalistes, mais alors, il nous faut aborder la situation des femmes autrement que comme le fruit d'une politique particulariste ; autrement que comme une politique destinée à fonder l'universel sur l'exclusion et la différence, c'est-à-dire sur ses propres contradictions.

C'est à cette contradiction que je me suis heurtée et qui a justifié mes premières recherches, au début des années 1990 : comment comprendre la situation des femmes selon les catégories de l'époque, c'est à dire en prenant au sérieux l'universalisme révolutionnaire? Sur quelle base les législateurs ont-ils pensé une représentation nationale qui ne reconnaissait la capacité politique qu'à une toute petite partie de la nation, celle des électeurs ? Comment était assuré, dans ce cas, le lien entre le représenté-non électeur et le représentant? Entre le fait d'être partie de l'universel, et le fait d'être « exclu» du droit de suffrage ?

On sait, depuis les travaux de Lynn Hunt, avec quelle force les figures du père et de la mère traversent l'imaginaire politique de la période révolutionnaire ; Suzanne Desan, quelques années plus tard, a montré comment les lois de la famille ont obéi, de 1789 au Code civil inclus, à des impératifs d'ordre public : le mariage laïc, la loi sur le divorce, sur l'égalité successorale entre frères et sœurs, sur l'adoption... sont parmi les lois qui suscitent le plus de débats et constituent les enjeux 
politiques les plus forts de la décennie révolutionnaire. Jennifer Heuer, à sa suite, a mis au jour l'intrication entre les lois de la famille, de la citoyenneté et de la nationalité. Pour ma part, j'avais commencé par un travail sur les lois électorales depuis l'an X jusqu'à la veille de 1848 qui m'avait amenée à faire l'hypothèse d'une conception familialiste du suffrage pour la période révolutionnaire. Depuis, j'ai pu prolonger ces recherches et montrer que dès 1789 , les révolutionnaires dessinent euxmêmes les frontières de la famille politique : je veux dire par là qu'ils décident de la définition des solidarités familiales qui vont, ou non, jouer dans l'obtention du droit électoral; notamment en décidant que les époux seront toujours solidaires, alors même que c'est madame qui paie des impôts et non pas monsieur ; et que de ce fait, l'époux d'une commerçante qui paie une patente pourra se prévaloir des impôts de sa femme pour obtenir l'inscription sur les listes électorales ; ou en accordant à certains «fils de famille »-qui, sous puissance paternelle, ne paient pas d'impôt - la possibilité de contourner la règle électorale pour devenir électeurs.

En cherchant à comprendre ce qui unit politiquement les électeurs au reste de la nation, on perçoit le rôle politique qu'a joué cette organisation familiale des droits. En tant qu'unité politique, elle induit, à l'instar de la République, une indivisibilité des opinions et des intérêts de ses membres; comme le mariage est censé unir des cœurs et des corps, des familles et des patrimoines. Ce n'est donc pas la classe de sexe qui induit les positions des uns et des autres puisque tous les hommes n'ont pas le droit de voter. La notion de classe de sexe, qui n'est pas absente mais qui est largement minorisée, n'est pas capable d'unir, au niveau politique, ceux qui votent et ceux qui ne votent pas. Or, l'union et l'indivisibilité sont, comme chacun sait, au fondement de la pensée républicaine.

\section{Martine LAPIED}

Les méthodes utilisées par les femmes dans les deux camps : prises de parole, actions conflictuelles, surveillance et témoignages, mais aussi utilisation des lieux de sociabilité révolutionnaire, ostentation ou invisibilité, peuvent-elles être considérées comme relevant d'une forme de "créativité » féminine?

Dans quelle mesure la radicalité de ces positions, leur aspect hors normes, ont-elles été transcrites dans des représentations, des sensibilités, des émotions, voire des généralisations, influençant ainsi les réflexions ultérieures sur la place des femmes dans le champ politique? 


\section{Anne VerJus}

Il y a certes eu des actions radicales autant que conflictuelles, parfois propres aux femmes lorsque cela engageait des problématiques jugées comme féminines. Mais il y a surtout eu une tendance générale à nier le caractère politique des actions qu'elles pouvaient mener, du fait même qu'elles étaient menées par des femmes ; c'est en partie aussi ce qui en a sauvé un bon nombre de l'échafaud.

\section{Lynn Hunt}

Pour moi, l'une des étapes les plus importantes dans la prise de conscience des femmes tient à leur capacité, en combinant formes anciennes et nouvelles de l'action collective, à se rendre visibles au sein de l'espace politique. Pourquoi Edmund Burke est-il si exaspéré par les journées d'octobre ? Précisément à cause de cette combinaison qui permet aux femmes de devenir les actrices évidentes d'un événement politique crucial. Elles avaient toujours été aux avant-postes lors des émeutes populaires en faveur de la taxation, mais il s'agit là d'une manifestation qui se transforme en assaut du peuple en armes contre le centre même du pouvoir (ce qui était alors virtuellement inconcevable), et qui se conclut, avec le retour de la famille royale à Paris, en triomphe romain pour le peuple et en humiliation pour la monarchie. Et les femmes tiennent le premier rôle à chaque étape du parcours. Aussi, de ce jour, l'image de l'action féminine (avec la menace de violence) est associée à la radicalité du processus révolutionnaire lui-même. La prééminence des citoyennes relève du monde à l'envers. Les rassemblements de femmes, que ce soit sur les marchés, dans les clubs, au sein des groupes envahissant les assemblées, font peur, même aux leaders révolutionnaires, pour leurs aspects à la fois ataviques et futuristes.

\section{Dominique Godineau}

Sur de nombreux points (surveillance et force de la parole dans l'espace public, émeutes), les femmes ont utilisé des modes traditionnels d'actions féminines, mais dans un espace politique nouveau, qu'elles (ou partie d'entre elles) avaient pénétré et dont elles savaient utiliser les ressources et la langue. Lors de la crise de l'an III, des émeutières parisiennes justifient par exemple leur action par les droits déclarés en 1789: des manifestantes réclament du pain en brandissant la Table des froits de l'homme en tête de cortège (mars 1795) ou, ulcérées par la famine, mettent « le comité [de la section] en arrestation au nom du peuple souverain et de la loi » (avril 1795); et des émeutières de Prairial assurent : « Nous 
sommes le Souverain ». Limitées par leur exclusion de l'exercice des droits politiques et des organisations qui y étaient liées (assemblée générale, garde nationale), les plus engagées ont « profité » de toutes les potentialités pour agir et se faire entendre. Elles ont ainsi développé des formes d'action originales qui, sans leur être nécessairement propres, caractérisaient leurs pratiques: par exemple, leur présence remarquée dans les tribunes des clubs et des assemblées, d'où le peuple « surveillait » ses représentants - et dont ces « habituées des tribunes » (surnom des militantes) furent spécifiquement exclues après Prairial. Les nombreuses pétitions envoyées à l'Assemblée, par des femmes comme par des hommes, montrent aussi qu'elles se saisirent de tous les moyens pour affirmer une identité de citoyennes. Tout comme le soulignent la création de clubs, ou plusieurs revendications qui mêlent soutien à la Révolution et désir d'être reconnues dans l'espace politique - telles celles du port des armes (un des droits politiques du citoyen), ou de la cocarde (symbole de citoyenneté rendu obligatoire pour les femmes en septembre 1793 suite aux demandes des militantes révolutionnaires) -. Ou encore les adresses collectives de femmes assurant à la Convention à l'été 1793 que, bien qu'on ne leur ait rien demandé et que leur vote n'ait aucun poids légal, elles aussi se sont réunies et acceptent la Constitution « présentée à la sanction du peuple souverain ». D'une certaine façon, tous ces gestes (et d'autres) peuvent être vus comme une forme de «créativité », qui adapte le traditionnel au nouveau contexte, ou qui contourne l'exclusion pour reconstruire du politique.

Furent-ils pour autant particulièrement radicaux ? ou est-ce parce qu'ils provenaient de femmes qu'ils furent ainsi présentés par leurs adversaires politiques (révolutionnaires ou contre-révolutionnaires), sur fond d'affrontements, pour se figer ensuite au XIX ${ }^{\mathrm{e}}$ siècle?

\section{Jean-Clément MARTiN}

Il ne faudrait pas que la distinction de genre homme/femme s'ajoute aux distinctions biologiques, créant de nouvelles essences. Les acteurs les plus impliqués dans cette réflexion étaient conscients des enjeux globaux, comme en témoigne la phrase souvent citée de Romme, parlant des « hommes de l'un ou l'autre sexe ». La bizarrerie de la formule traduit un questionnement qui n'a toujours pas trouvé de solution aujourd'hui, puisque nous continuons à buter sur l'indécision du vocable « homme». La naturalité des comportements n'est pas fixée. Les capacités sociales d'agir sont liées aux positions sociales, inscrites dans les rapports de genre, et demeurent ouvertes aux initiatives, ce qui ouvre d'autres perspectives de recherches. 
Peut-on alors, au risque de durcir les traits, proposer une vue cavalière de la période au prisme du «genre» (et non plus seulement de l'histoire des femmes) ? L'inégalitarisme de la société d'ordre, accordant des libertés aux femmes, se maintient notamment en Vendée, où des femmes peuvent commander militairement, en remplacement d'hommes défaillants. Les mœurs libertines des émigrés se prolongent quelques années, avant de muter pour correspondre au conservatisme social qui sera le programme politique du royalisme. La société machiste, inégalitaire, devient le modèle commun des partisans de la Révolution, admettant, en guise de soupapes de sécurité, des transgressions, quand elles sont limitées dans leurs effets et exploitables par les hommes dominants. Les sans-culottes se font particulièrement remarquer avec leurs discours cantonnant les femmes derrière les fourneaux et dans les lits. Les girondins, plus tard les Idéologues, ont des positions plus libérales, voire complexes, tandis que certains individus comme Condorcet, Guyomar, prônent l'égalité entre hommes et femmes. Dans les marges, des communautés religieuses ou sectaires mènent des expériences originales tandis que des démarches plus «égalitaires » sont le fait d'individus comme Romme, ou d'une partie des citoyennes révolutionnaires et des Enragés.

\section{Martine LAPIED}

Au-delà de la focalisation sur la question des droits politiques, peut-on s'interroger sur le rôle de la Révolution pour les femmes, sur les champs qui leur sont, ou non, ouverts par la rupture révolutionnaire? En effet, les évolutions des analyses peuvent être liées à différentes méthodes d'étude, à des questionnements élargis. Politisation de l'espace privé, questions sociales, question religieuse revisitée, prise en compte des trajectoires individuelles, approches micro-historiques, ancrages des recherches dans le local... Quelles vous semblent être les voies fructueuses dans les questionnements actuels?

\section{Lynn HunT}

Toutes ces approches me semblent fructueuses. En effet, la plupart d'entre elles (exceptées la micro-histoire et les monographies locales) ont été soit initiées soit revitalisées par ceux qui ont étudié l'histoire des femmes et du genre. J'ai le sentiment que nous allons voir sous peu grossir l'intérêt pour le rôle des femmes esclaves dans l'économie de la traite et dans la révolution haïtienne (Elizabeth Colwill, de l'Université de San Diego State, travaille sur le sujet), tout autant que pour leur rôle dans le développement économique général de la fin du XVIII ${ }^{\mathrm{e}}$ siècle, aussi bien 
comme consommatrices (de thé, de café, de chocolat, d'épices, de chinoiseries, de vêtements de coton, etc...) que comme productrices ou vendeuses de nouveaux produits. Il est important de constater que le rôle des femmes dans ces deux cas (l'esclavage, l'économie globale) ne devient apparent que si l'on développe la micro-histoire ou une recherche ancrée dans le tissu local, d'où l'importance de ces méthodes. Plus l'approche est localisée, plus la reconnaissance de la participation féminine est reconnue. Le risque d'une histoire de plus en plus globalisée est au contraire de la maintenir dans l'ombre, au profit des habituels thèmes de la diplomatie, de la guerre, du commerce au long cours, domaines dans lesquels les femmes sont traditionnellement réduites au rôle de spectatrices (au mieux, dans le cas des études militaires, à celui de cantinières).

\section{Anne VerJus}

Les recherches qui sont en cours montrent que l'intérêt pour une prise en compte des catégories de pensée de l'époque a orienté les historiennes vers des terrains jusqu'alors délaissés, notamment en raison de leur ancrage traditionnel dans le masculin : sur celui des guerres napoléoniennes, et notamment des politiques matrimoniales qui les ont soutenues, Jennifer Heuer travaille actuellement à comprendre les liens entre mariage et politique ; les prêtres également sont étudiés actuellement dans le cadre d'une thèse sur l'incitation au mariage comme politique républicaine (Claire Cage) ; Suzanne Desan et Jeffrey Merrick, dont on connaît l'intérêt pour la famille comme idéologie, ont publié un collectif dont l'objet est précisément l'articulation juridique du familial et du genre. Tout un ensemble de recherches, on le voit, sont en cours qui examinent les implications politiques, sociales, juridiques et économiques d'une société articulée autour du familial et des hiérarchies qui en découlent.

\section{Jean-Clément MARTiN}

Sexe, genre et sexualités sont au cœur du processus révolutionnaire : les remises en cause des hiérarchies femmes/hommes qui se produisent, sont les signes de la révolution culturelle sous-jacente qui a alimenté la radicalité politique. Dans une société très hiérarchisée, les contestations sont passées initialement par des biais culturels ou religieux. Le moment du bouleversement révolutionnaire leur a donné ensuite une coloration politique qui en a altéré le sens et créé des interprétations destinées ensuite à durer.

L'histoire de la Révolution a créé de nouvelles normes, remplaçant celles qui existaient auparavant. Or tous ces renouvellements étant étroi- 
tement liés les uns les autres, la signification qui en résulte excède les découpages ordinaires utilisés par les historiens recourant aux domaines politique ou social, ou aux notions d'espaces public et privé. S'intéresser aux relations de genre permet de comprendre l'unité profonde qui joue sous ces distinctions et les conteste. Par exemple, l'espace intime d'un appartement parisien est à l'évidence en interaction avec l'espace public de la rue et du quartier. L'analyse par le genre rend visibles les lignes de fracture qui ont affaibli la société française, et souligne les divisions exploitées par la lecture politique née des événements.

Plus que faire une histoire « des femmes », il s'agit là de rendre compte d'un moment créant des identités collectives, auxquelles chacun est sommé de se conformer. Le «muscadin » contre-révolutionnaire est évidemment parfumé et efféminé. Les soldats seront identifiés par la moustache, et plus aucun d'entre eux ne sera jamais habillé en rose, comme l'étaient les troupes du prince de Ligne, attaché à cette couleur. L'uniforme noir des hommes et les épaules nues des femmes seront les repères de la cour de Napoléon ${ }^{\mathrm{I} r}$. Il serait temps que le portrait régulièrement dressé du sans-culotte, identifié par son bonnet rouge et sa pique, ne soit plus pris au pied de la lettre par les historiens, acceptant d'être attentifs aux dimensions fantasmatiques, qui façonnent les sociétés.

Dans ces relations de genre, révélateurs et occasions de tensions, l'exemple le plus significatif est certainement la législation sur le divorce. Votée en 1792 dans la suite de la valorisation de l'amour conjugal, elle devient l'emblème paradoxal de la libération des femmes provoquée par la Révolution, et est dès 1795 l'objet d'un dénigrement qui aboutira à son annulation. Le cœur du social est ici mis à nu par les innovations liées au droit de la famille, puisqu'en 1794 les enfants naturels ont les mêmes droits que les enfants légitimes. Le reflux des mentalités ne se fait ici que dans les années 1795-1796. À ce moment, la fascination envers les « merveilleuses »-comme la mutation de la littérature pornographique - trouve sa signification politique ; leur évocation permet de condamner la Terreur, qui a brimé la vie sociale, perturbé les hiérarchies sociales et libéré les pulsions. Si bien que le miroir déformant tendu soigneusement par les vainqueurs de Thermidor, pour accabler Robespierre, n'en finit pas de contraindre l'écriture de l'histoire et la transmission mémorielle. La politisation du pays était passée davantage par les sensibilités, liées au genre, que par la diffusion des idées des Lumières; les résonances des événements révolutionnaires sont souvent nées, par la suite, en écho aux récits des atrocités commises contre les femmes, achevant le processus tout en le détournant. 


\section{Dominique Godineau}

Il est effectivement important de ne pas oublier que la législation révolutionnaire a modifié le statut civil et familial des femmes. Des mineures juridiques de l'Ancien Régime, elle a fait des individus majeurs possédant pleine capacité juridique, a instauré l'égalité successorale et le divorce, donnant aux Françaises le statut le plus progressiste du monde occidental - ce qui fut d'ailleurs dénoncé avec vigueur par des théoriciens de la contre-révolution (Burke) et amène à relativiser la misogynie des révolutionnaires. Allant plus loin que cette simple mise au point, Suzanne Desan a récemment démontré comment les femmes se sont approprié cette législation, et son travail me paraît emblématique des chemins ouverts par les recherches actuelles.

De nombreux thèmes peuvent ainsi être réexaminés (et ont déjà commencé à l'être) sous l'angle du genre, selon la démarche présentée plus haut, en croisant analyses des pratiques, des discours, des représentations ou de la législation.

La richesse et l'inventivité des travaux qui, quoique de façon différente, abordent le politique par le prisme de la famille incitent à ne surtout pas abandonner cet axe familial. De même, est loin d'être épuisé celui qui consiste à analyser comment le «paradigme » (Lynn Hunt) genre (le masculin-féminin comme élément structurant d'une société et des représentations qu'elle donne d'elle-même) intervient et fonctionne dans la culture politique.

Dans une optique « histoire des femmes » ouverte aux problématiques actuelles, il faut absolument poursuivre les recherches ancrées dans le local, comme cela a pu être fait sur Paris ou le Sud-Est. Des pistes avaient été lancées lors du colloque de Toulouse (1989) ; des maîtrises ou masters dirigés dans plusieurs universités apportent d'intéressantes informations. Mais l'on est encore loin d'avoir une vue d'ensemble de la participation féminine (qu'elle soit pour ou contre la Révolution), qui semble fort contrastée suivant les lieux. Ces travaux ont par ailleurs fait ressortir l'importance de la question religieuse, qui bénéficie depuis quelques années d'un stimulant renouveau (sur les religieuses, les « dévotes contrerévolutionnaires », les formes prises par l'actif soutien féminin au clergé réfractaire, voire sur les tentatives de concilier sentiments religieux et soutien à la Révolution).

L'articulation entre le singulier et le général serait une autre piste à creuser : parallèlement à l'approfondissement des recherches sur les femmes ordinaires, l'exploration des marges (les femmes soldats, les héroïnes, etc...) est une démarche souvent féconde, qui révèle beaucoup 
sur le fonctionnement (normes, transgressions, négociations) et les tensions de la société révolutionnaire, à condition bien entendu de ne pas oublier qu'il s'agit de marges.

Enfin, trois entrées mériteraient, me semble-t-il, une plus grande attention. Tandis que l'historiographie accorde une place grandissante aux années 1770, notamment pour la formation d'une culture politique, cette voie n'est encore que peu empruntée en histoire du genre alors qu'elle s'avèrerait probablement très fructueuse. Le serait également un angle d'approche " guerre », dont les travaux sur les femmes dans les armées républicaines ou en Vendée ont montré l'intérêt, sans toutefois l'épuiser : que signifie vivre dans un pays en guerre et quelles en sont les répercussions sur la représentation des deux sexes comme sur le concret des vies ? Quels furent les effets économiques, sociaux, familiaux, politiques, culturels de l'absence de nombreux hommes (ou de la forte présence de soldats dans certaines régions, telle l'Ouest) ? Comment s'est manifesté le patriotisme en fonction du genre ? Enfin, je souhaiterais, à titre personnel, rouvrir le dossier du travail féminin, en l'abordant sous l'angle des rapports entre genre, travail, citoyenneté, culture politique et engagement.

\section{Martine LAPIED}

En individuant les femmes, donc en leur accordant reconnaissance et visibilité, modifie-t-on notre perception de l'ordre social mis en place au cours de la Révolution française, dans la mesure où leur présence genrée " trouble " cet ordre tant au niveau de la société politique que de la société civile (la famille principalement), et rend ainsi plus visible, en matière d'émancipation, la part commune avec les hommes?

\section{Anne VerJus}

L'invisibilité est, en effet, la première caractéristique des femmes sous la Révolution. Je préfère, pour ma part, ce mot à celui d'exclusion, car il renvoie à la non inclusion, à la non prise en compte (sinon en considération), par les législateurs, de cette partie de la population dont ils pensaient sincèrement connaître les intérêts, pouvoir assurer la protection, et savoir mieux que quiconque, finalement, ce qui était bon pour elles. Il faut se garder de la tentation individualisante qui nous amène à juger les femmes - et les opinions sur les femmes - à partir de nos propres lunettes ; nous faisons aux générations du temps passé un procès qui n'a pas lieu d'être. Penser une citoyenneté pour les femmes impliquait d'abord de les désencastrer du familial et de penser leurs intérêts indépendamment de 
ceux des hommes en tant que chefs de famille. Historiquement, il a fallu en passer par les intérêts et la solidarité des femmes en tant que classe de sexe avant de pouvoir envisager leur « part commune avec les hommes ", c'est-à-dire leur capacité à exprimer une opinion individuelle. Et c'est seulement à ce moment, d'ailleurs, que le vote exprimé par les hommes a été assumé par la majorité parlementaire, en miroir, comme un vote « de classe de sexe ». "Les législateurs font les lois pour ceux qui font les législateurs » avait déclaré Viviani avant d'emporter l'adhésion massive des députés au droit de vote des femmes en 1919 : voilà une expression et un principe que n'auraient pas revendiqués les révolutionnaires.

\section{Dominique Godineau}

Ces études soulignent surtout les paradoxes de la Révolution (et par conséquent son intérêt et sa richesse) car elles obligent à retravailler les catégories habituelles de pensée (sur les droits, l'exclusion, le politique...). Pour conclure, je dirais que, en accordant l'exercice des droits politiques aux seuls hommes, les révolutionnaires ont introduit une nouvelle inégalité ; mais en affirmant le principe fondateur d'égalité, ils ont rendu l'injustice plus flagrante et donné des armes pour la combattre armes que certain(e)s surent utiliser pendant la Révolution même.

\section{Lynn Hunt}

C'est moins leur présence « genrée », qui trouble l'ordre social ou politique, que la définition même de cet ordre social et politique. Une fois effondrées les fondations classiques du pouvoir (privilège, naissance, hiérarchie), tout est remis en cause. Parce qu'elle transforme radicalement la donne, la Révolution révèle le fait que l'ordre politique et social dépend de certaines attentes concernant les femmes. Comment ce nouvel ordre sera-t-il défini ? Quelles sont les différences entre hommes et femmes? Ces différences font-elles problème maintenant que le roi est simplement un homme comme les autres ? Font-elles encore plus question? Quelles différences comptent dans cette nouvelle organisation (la question vaut encore pour nous aujourd'hui !) ? Le simple fait de contraindre le peuple à s'interroger de la sorte conduit certains à répondre que les différences entre hommes et femmes, telles qu'elles existent, n'apparaissent pas pertinentes dans l'ordre politique (Condorcet, Olympe de Gouges, qui se contente de le suggérer). Cela amène la plupart des politiques, cependant, à plaider le maintien de la différence, bien qu'ils ne se soient pas étendus longtemps sur des raisons convaincantes (ayant déjà nié la biologie par respect de la noblesse et du roi, ils doivent travailler dur pour la convoquer 
dans ce cas précis). La différence entre hommes et femmes doit être une différence qui va s'en dire parce que beaucoup d'autres ont été contestées. Aussi, je considère l'exclusion des femmes comme une conséquence d'une peur inconsciente de l'éradication de toutes les différences.

\section{Jean-Clément MARTIN}

L'intérêt porté au genre, dit autrement à la construction sociale des identités sexuées, conduit donc à d'autres conclusions, plus fondamentales. Ainsi l'importance considérable de l'érotisation de la propagande révolutionnaire ou contre-révolutionnaire témoigne de l'interrogation collective portant sur les relations entre hommes et femmes à la fin du XVIII ${ }^{\mathrm{e}}$ siècle. La place assignée aux femmes dans les pamphlets, ou dans les fêtes, réinterprète politiquement les fantasmes récurrents des hommes. Ces derniers dénoncent leur pouvoir fondé sur la séduction, et craignent la concurrence qu'elles représentent au moment où eux-mêmes s'affranchissent de l'autorité nobiliaire. L'édification forcenée et inquiète de l'identité masculine est en cause. L'opprobre jeté sur la masturbation, l'insistance sur la virilité du patriote et du militaire, le refus du libertinage aristocratique - paradoxalement associé souvent à la «mollesse » de l'aristocrate - sont au cœur du projet de régénération sociale porté par toute la société de la fin du XVIII ${ }^{\mathrm{e}}$ siècle et assumé spécifiquement par les révolutionnaires après 1790-1791. Marie-Antoinette est l'exemple privilégié de cette fixation, ce que Olympe de Gouges ou Mme de Staël ont parfaitement compris, s'estimant concernées par le sort fait à la reine en tant que femme soumise à la loi sociale.

Sans entrer dans le débat existant aujourd'hui entre tenants du genre et ceux qui, relevant les « troubles » dans le genre, refusent le risque d'une nouvelle naturalité des catégories et insistent sur les apports de la théorie queer (qui met en avant les « performances », les « incertitudes »), force est de penser que cette dernière lecture éclaire aussi ce qui s'est passé pendant la Révolution. Il n'y a aucun hasard dans le fait que le/la chevalier/ère d'Eon cesse d'être un objet de scandale et d'intérêt, et est relégué/e dans un espace purement privé au moment de la Révolution. Le « trouble dans le genre » que l'on peut constater dans sa réalité vécue au moment de la Révolution française, correspond bien aux indécisions qui s'attachent aux identités personnelles et collectives, et que la Révolution va s'attacher à éliminer, en reclassant hommes et femmes. L'inquiétude avant 1789 s'alimentait à tous ces brouillages qui existaient dans la société déréglée illustrée par des nobles libertins, des servantes maîtresses, des écrivaines demi-mondaines, voire par une reine fermière ; comme l'écrit 
Robert Darnton, « le XVIII ${ }^{\mathrm{e}}$ siècle ne cesse de se déguiser et de changer de costumes ». Théroigne de Méricourt est alors exemplaire pour le rôle qu'elle joue dès 1789 , comme pour la répression qu'elle subit, hommes et femmes révolutionnaires hostiles à cette aventurière brouillant les distinctions.

Les réflexions sur les stratégies queer comme recherche d'une vérité personnelle, ou au moins d'expression individuelle, allant jusqu'au sacrifice et à l'ascèse, permettent alors de comprendre les itinéraires individuels pendant le moment exceptionnel de la Révolution. Cette approche combinée avec d'autres analyses sociologiques, comme celles qui accordent toute leur importance aux « outsiders » si nombreux et si importants, rendent compte des attitudes des femmes jansénistes à Paris ou dans le Lyonnais, comme celles des catholiques allant à l'échafaud dans l'Ouest. La période révolutionnaire aura été par excellence le temps de la libération des énergies individuelles et collectives, excédant le cadre politique convenu. Tous les historiens butent sur les personnages « excessifs » que la Révolution libère et qui sont les auteurs, éventuellement involontaires, des « effervescences » propres à toute rupture radicale. Par la suite, l'Empire canalisera cette énergie surnuméraire, qui avec la Restauration et la Monarchie de Juillet ne pourra s'investir que dans la littérature, la religion officielle ou déviante, bref l'espace privé - avant les exutoires coloniaux ou les espaces transgressifs.

Dominique GodinEaU

Université Rennes 2

CERHIO UMR 6258

domgodineau@wanadoo.fr

Martine LAPIED

Université de Provence (Aix-Marseille I)

UMR TELEMME - MMSH

lapied@mmsh.univ-aix.fr
Lynn HunT

Department of History

UCLA (Los Angeles, CA, USA)

lhunt@history.ucla.edu

Jean-Clément MARTIN

Université Paris 1 - IHRF

jean-clement.martin@orange.fr

Anne VerJus

CNRS, Université de Lyon, Ens-Lsh

UMR 5206 Triangle

Anne.verjus@ens-lsh.fr 